\title{
IMAGEM CORPORAL E FATORES ASSOCIADOS EM MENINAS DE ESCOLAS MILITARES
}

\section{Body image and associated factors in girls of military schools \\ Imagen corporal y factores asociados en las niñas de escuelas militares}

\author{
Felipe Rocha Alves \\ Universidade Federal do Ceará - UFC - Fortaleza (CE) - Brasil
}

\section{Francisca Érica de Lima Bezerra}

Universidade Estadual do Ceará - UECE - Fortaleza (CE) - Brasil

Evanice Avelino de Souza

Universidade Federal do Ceará - UFC - Fortaleza (CE) - Brasil

\section{Francisco Alex Alves Teixeira}

Faculdade Terra Nordeste - FATENE - Caucaia (CE) - Brasil

\section{RESUMO}

Objetivo: Investigar a prevalência de insatisfação com a imagem corporal (IC) em adolescentes do sexo feminino e sua associação com faixa etária, estado nutricional e prática de atividade física. Métodos: Estudo transversal, realizado com 393 adolescentes do sexo feminino (entre 14 e 18 anos) das escolas militares da cidade de Fortaleza, Ceará, no período de agosto a novembro de 2015. Utilizou-se a escala de silhuetas de Stunkard para a avaliação da IC. O estado nutricional foi avaliado segundo o Índice de Massa Corporal (IMC) $(\mathrm{IMC}=$ peso/ estatura $^{2}$ ) e a prática de atividade física (sim ou não) foi autorrelatada. Inicialmente, recorreu-se à análise descritiva em valores absolutos (n) e relativos (\%), em seguida empregou-se o teste do Qui-quadrado, adotando-se $p \leq 0,05$. Resultados: A maioria tinha entre 16-17 anos de idade $(52,7 \%, \mathrm{n}=207)$, praticava atividade física $(62,6 \%, \mathrm{n}=246)$, apresentava peso adequado $(63,4 \%, \mathrm{n}=249)$ e estava insatisfeita com a IC $(65,4 \%, n=257)$. A faixa etária e a prática de atividade física estavam associadas à $\mathrm{IC}(\mathrm{p}=0,04 \mathrm{e} \mathrm{p}=0,02$ respectivamente). Não se constatou associação do estado nutricional com a IC nessa amostra. Conclusão: Os resultados indicam uma elevada prevalência de insatisfação com a imagem corporal, independente da faixa etária e da prática de atividade física.

Descritores: Percepção; Imagem corporal; Adolescentes.

\section{ABSTRACT}

Objective: To investigate the prevalence of body image (BI) dissatisfaction in female adolescents and its association with age range, nutritional status and physical activity practice. Methods: A cross-sectional study of 393 female adolescents (aged 14-18 years) studying in military schools of the city of Fortaleza, Ceará, in the period from August to November 2015. The figure rating scale by Stunkard was used for BI assessment. The nutritional status was evaluated according to the Body Mass Index (BMI) (BMI = weight/height $\left.{ }^{2}\right)$ and the practice of physical activity (yes or no) was self-reported. Descriptive analysis was initially conducted, in absolute (n) and relative (\%) values, followed by the chi-square test, adopting $p \leq 0.05$. Results: A majority of the adolescents were 16-17 years old $(52.7 \%, n=207)$, practiced physical activity $(62.6 \%, n=246)$, adequate weight $(63.4 \%, n=249)$ and were dissatisfied with their BI $(65.4 \%, n=257)$. Age range and physical activity were associated with BI ( $p=0.04$ and $p=0.02$, respectively). There was no association between nutritional status and BI in this sample. Conclusion: The results indicate a high prevalence of body image dissatisfaction, regardless of age group and physical activity practice.

Descriptors: Perception; Body image; Adolescents 


\section{RESUMEN}

Objetivo: Investigar la prevalencia de insatisfacción de adolescentes del sexo femenino con el imagen corporal (IC) y su asociación con la franja de edad, el estado nutricional y la práctica de actividad física. Métodos: Estudio transversal realizado con 393 adolescentes del sexo femenino (entre los 14 y 18 años) de las escuelas militares de la ciudad de Fortaleza, Ceará, en el periodo entre agosto y noviembre de 2015. Se utilizó la escala de siluetas de Stunkard para la evaluación del IC. El estado nutricional fue evaluado a través del Índice de Masa Corporal (IMC) (IMC= peso/altura ${ }^{2}$ y la práctica de actividad física (sí o no) fue auto relatada. A principio se realizó un análisis descriptivo en valores absolutos (n) y relativos (\%) y en seguida se utilizó la prueba de Chi-cuadrado con el p $\leq 0,05$. Resultados: La mayoría tenía entre los 16-17 años de edad (52,7\%, $n=207)$, practicaba actividad fisica (62,6\%, $n=246)$, tenía el peso adecuado $(63,4 \%, n=249)$ y estaba insatisfecha con el IC $(65,4 \%, n=257)$. La franja de edad y la práctica de actividad física se asociaron al IC $(p=0,04$ e p=0,02 respectivamente). No se ha constatado asociación entre el estado nutricional y la IC de la muestra. Conclusión: Los resultados indican una elevada prevalencia de insatisfacción con la imagen corporal independiente de la franja de edad y de la práctica de actividad fisica.

Descriptores: Percepción; Imagen corporal; Adolescente.

\section{INTRODUÇÃO}

A imagem corporal (IC) pode ser definida como a percepção que o individuo tem do próprio corpo de acordo com as vivências e sensações experimentadas durante sua vida ${ }^{(1)}$. Nas últimas décadas, têm se vivenciado uma mudança em relação aos padrões de beleza impostos pela sociedade. Observa-se uma crescente preocupação com a IC, especialmente na busca por um padrão de beleza idealizado ${ }^{(2)}$.

Bastante evidente durante a adolescência, a insatisfação corporal atinge principalmente indivíduos do sexo feminino ${ }^{(3)}$. A forma como o adolescente percebe sua IC traz consequências para sua saúde física e mental, com possíveis repercussões em suas relações pessoais ${ }^{(4)}$.

A insatisfação corporal está associada a sintomas depressivos, estresse, baixa autoestima, maior restrição alimentar, além de passar por uma valorização cultural que se modifica de acordo com o gênero, o estado nutricional e a prática de atividade física $^{(5)}$.

O estudo sobre a insatisfação corporal e potenciais fatores associados em adolescentes é de suma importância por possibilitar a identificação de fatores que podem ser modificados ${ }^{(6)}$, por isso crescente espaço na literatura têm sido dado à temática da insatisfação corporal entre adolescentes, com concentração de estudos nas Regiões Sul e Sudeste do Brasil ${ }^{(7,8)}$. $\mathrm{O}$ conhecimento da dinâmica da adolescência em relação ao seu corpo, às influências externas e às características associadas são fundamentais para o reconhecimento precoce e a adoção de medidas preventivas de distúrbios da IC. Além de tornar mais eficazes os programas de prevenção e combate aos problemas relacionados à insatisfação corporal nessa população( ${ }^{(9)}$.

Nesse contexto, a escola apresenta-se como o ambiente ideal para programas de intervenção, pois reuni periodicamente um grande número de adolescentes em seus turnos de estudo. Nesse sentido, o objetivo deste estudo foi investigar a prevalência de insatisfação com a imagem corporal (IC) em adolescentes do sexo feminino e sua associação com faixa etária, estado nutricional e prática de atividade física.

\section{MÉTODOS}

O estudo apresentou delineamento transversal e ocorreu entre agosto e novembro de 2015. A pesquisa envolveu adolescentes do sexo feminino, regularmente matriculadas no $3^{\circ}$ ano do ensino médio das escolas militares da cidade de Fortaleza, Ceará, Brasil. Como critério de inclusão, tinha-se que estar matriculada no $3^{\circ}$ ano do ensino médio; e, como critério de exclusão, as que não entregaram Termo de Consentimento Livre e Esclarecido (TCLE) assinado pelos pais e/ou responsáveis.

Primeiramente, entregou-se às coordenações das escolas o termo de anuência para a autorização do estudo. Não havendo oposição dos coordenadores em relação à realização do estudo nas instituições, agendou-se uma data para a apresentação da pesquisa às adolescentes (exposição dos objetivos e relevância do estudo, e esclarecimento de possíveis dúvidas). Em uma terceira visita às instituições, iniciou-se a coleta de dados com as adolescentes que estavam presentes nos dias de coleta.

A coleta de dados foi realizada por estudantes do curso de Educação Física, pertencentes ao Grupo de Pesquisa em Saúde e Atividade Física na Escola (UFC), após treinamento teórico-prático e realização de coleta supervisionada no estudo piloto.

A percepção da IC foi analisada pela escala de nove silhuetas, proposta por Stunkard et al. ${ }^{(10)}$, a qual representa um continuum desde a magreza (silhueta 1) até a obesidade severa (silhueta 9). Nessa escala, o indivíduo escolhe o número da silhueta que considera semelhante à sua aparência corporal real (Percepção da IC Real - PICR) e também o número da silhueta que acredita ser mais condizente a sua aparência corporal ideal (Percepção da IC Ideal - PICI). Para a avaliação da satisfação corporal, subtraiu-se da aparência corporal real a aparência corporal ideal, podendo esse número variar de -8 até +8 . Caso essa 
variação fosse igual a zero, a adolescente era classificada como satisfeita com sua aparência e, se diferente de zero, classificavase como insatisfeito. Caso a diferença fosse positiva, considerava-se uma insatisfação pelo excesso de peso e, quando negativa, uma insatisfação pela magreza.

As medidas de peso e altura foram mensuradas diretamente, utilizando-se (i) balança digital da marca Plenna ${ }^{\circledR}$, com capacidade para $150 \mathrm{~kg}$ e precisão de $100 \mathrm{~g}$, e (ii) fita métrica metálica inextensível, de $150 \mathrm{~cm}$ de comprimento e precisão de 1,0 mm, afixada a um metro de altura, sobre uma parede lisa. Mensuraram-se as adolescentes sem calçados, com o mínimo de roupas possível, em sala reservada. O estado nutricional foi avaliado segundo o Índice de Massa Corporal (IMC) (IMC $=$ peso/ estatura $^{2}$ ). A classificação das adolescentes nas categorias de IMC foi baseada nos pontos de corte proposto para adolescentes ${ }^{(11)}$ até 17 anos e pela Organização Mundial de Saúde (OMS) para as adolescentes maiores de $18 \operatorname{anos}^{(12)}$.

A variável (faixa etária) era autorrelatada e a prática de atividade física obtida mediante a seguinte pergunta: Você prática atividade física extracurricular? (sim ou não).

Para análise dos resultados, utilizou-se estatística descritiva em valores absolutos (n) e relativos (\%). O teste do Qui-quadrado foi utilizado para a análise das variáveis (estado nutricional, faixa etária e prática de atividade física) com a classificação da IC. O nível de significância foi estabelecido em $\mathrm{p} \leq 0,05$ para as análises finais. Todos os dados foram analisados através do software IBM SPSSR Statistics 21.0.

O protocolo do estudo recebeu aprovação pelo Comitê de Ética em Pesquisa da Secretaria de Saúde do Estado do Ceará SES/CE (Parecer n 1232078/2015), respeitando a Resolução 466/12 do Conselho Nacional de Saúde.

\section{RESULTADOS}

Todas as adolescentes $(\mathrm{n}=494)$ matriculadas no $3^{\circ}$ ano do ensino médio foram convidas a participar do estudo, porém algumas não fizeram parte $(\mathrm{n}=81)$ porque não entregaram o TCLE assinado pelos pais e/ou responsáveis e/ou porque não estavam presentes no dia da coleta $(\mathrm{n}=21)$. Dessa forma, a amostra final do estudo foi composta por 393 adolescentes distribuídas da seguinte forma: Colégio Militar do Corpo de Bombeiros ( $\mathrm{n}=98$ ); Colégio da Policia Militar ( $\mathrm{n}=134$ ) e Colégio do Exército $(n=161)$.

A Tabela I faz uma descrição das adolescentes das escolas militares de Fortaleza, Ceará, e mostra que a maioria das participantes tinha entre 16 e 17 anos, praticava atividade física, foram classificadas com peso adequado e estavam insatisfeitas com a IC.

Em relação à classificação da $\mathrm{IC}$, os resultados indicaram que as adolescentes maiores de 18 anos $(42,9 \%, \mathrm{p}=0,04)$ e que praticavam atividade física $(43,5 \%, p=0,02)$ apresentaram maior insatisfação por excesso de peso. Não houve associação do estado nutricional com a IC. (Tabela II.)

Tabela I - Características gerais da amostra em relação à faixa etária, prática de atividade, estado nutricional e percepção corporal das adolescentes em valores absolutos (n) e relativos (\%), Fortaleza, Ceará, 2015 (n=393).

\begin{tabular}{lcc}
\hline Variáveis & $\mathbf{n}$ & $\mathbf{\%}$ \\
\hline Faixa etária & 158 & 40,2 \\
$14-15$ & 207 & 52,7 \\
$16-17$ & 28 & 7,1 \\
$>18$ anos & 246 & 62,6 \\
Prática de atividade física & 147 & 37,4 \\
$\quad$ Sim & & \\
Não & 249 & 63,4 \\
Classificação nutricional & 109 & 27,7 \\
$\quad$ Adequado & 31 & 7,9 \\
Excesso de peso & 4 & 1 \\
Obesidade & & \\
Baixo peso & 136 & 34,6 \\
Percepção corporal (diferença na escala de silhuetas) & 257 & 65,4 \\
Satisfeitas & 93 & 23,7 \\
Insatisfeitas & 164 & 41,7 \\
$\quad$ Insatisfeitas por magreza & & \\
Insatisfeitas por excesso de peso &
\end{tabular}


Tabela II - Valores absolutos (n) e relativos (\%) para a classificação da imagem corporal e associação com estado nutricional, faixa etária e prática de atividade física, Fortaleza, Ceará, 2015 (n=393).

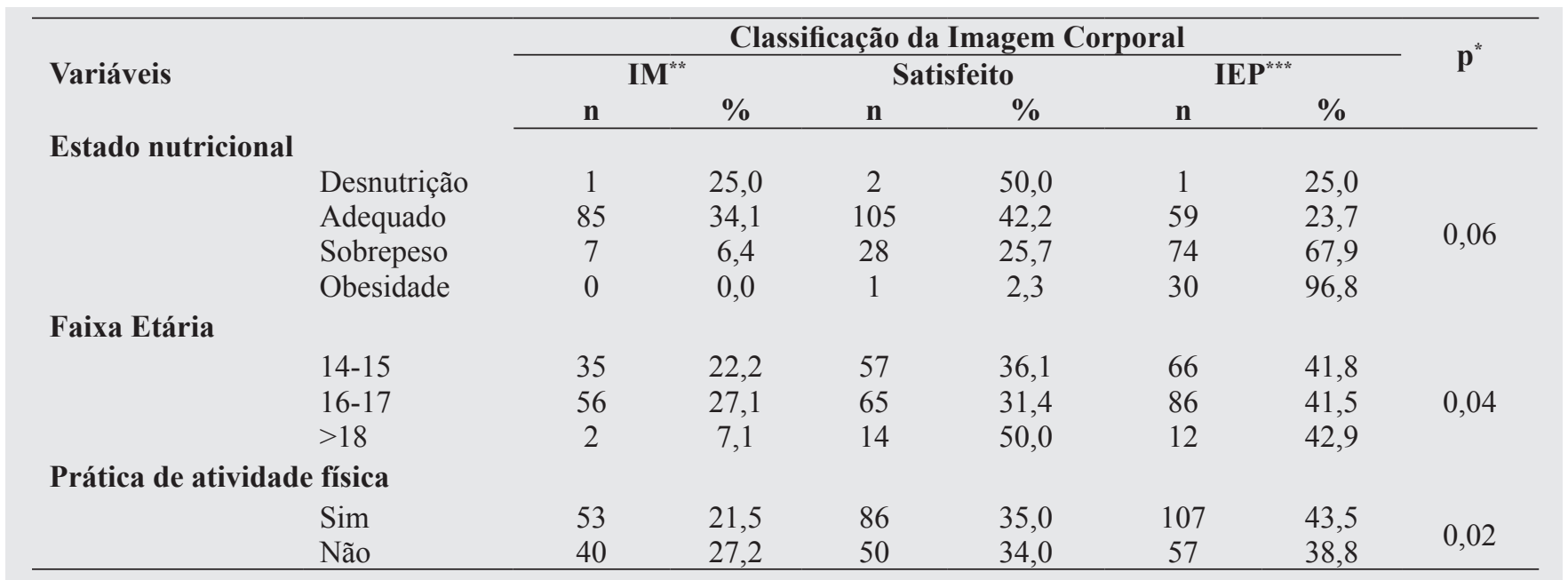

* Teste de Qui-quadrado; ${ }^{* *}$ IM: insatisfeito por magreza; ${ }^{* * *}$ IEP: insatisfeito por excesso de peso

\section{DISCUSSÃO}

O estudo sobre insatisfação corporal em adolescentes possibilita a identificação de aspectos modificáveis. As respostas produzidas poderão orientar profissionais da saúde e educação na elaboração de programas de prevenção e combate aos problemas associados à insatisfação corporal nessa população. O presente estudo registrou uma prevalência de insatisfação com a IC. A maioria das adolescentes estava insatisfeita por excesso de peso (IEP), resultado que é bem descrito na literatura ${ }^{(9,13,14)}$. Enquanto elas desejam reduzir o tamanho da silhueta corporal, os adolescentes do sexo masculino desejam aumentar. Reforçando essa afirmação, um estudo conduzido em escolas públicas da cidade de Santa Maria, Rio Grande do Sul, com 1.126 adolescentes, assim como outro estudo, realizado na cidade de Irati/PR, com 340 adolescentes, registraram resultados semelhantes ao do presente estudo ${ }^{(4,15)}$.

Entre os fatores que explicam a prevalência de insatisfação com a IC entre adolescentes do sexo feminino, está a elevada cobrança social e a forte influência da mídia pela busca do corpo perfeito, podendo se alastrar para a vida adulta ${ }^{3,16}$.

Existe uma facilidade de acesso aos veículos midiáticos, especialmente àqueles relacionados às redes sociais, sendo a internet um potente meio sociocultural que contribui para a distorção da IC, a que os adolescentes estão diariamente expostos, tornando-se um grupo de risco ${ }^{(17)}$.

Atrelados a esse contexto, o padrão de beleza estabelecido pela sociedade passou por substanciais mudanças nos últimos anos, com a propagação da atratividade representada como magreza para as mulheres e corpo musculoso para os homens ${ }^{(18)}$. O sobrepeso tornou-se alvo de discriminação em vários locais, sendo rotulado como sinal de preguiça, falta de disciplina e motivação $0^{(19)}$. Os padrões de beleza são onipresentes e estendem-se a toda a população, além de serem inatingíveis pela maioria das pessoas. Assim, o conhecimento da percepção que o adolescente tem do seu próprio corpo, as influências externas e os fatores associados são fundamentais para o reconhecimento precoce e a adoção de medidas preventivas de distúrbios da IC ${ }^{(20)}$.

Os resultados do presente estudo não demonstraram associação do estado nutricional com a IC, entretanto verificou-se que $67,9 \%$ das adolescentes classificadas com sobrepeso estavam IEP. O desejo por uma silhueta menor do que a atual têm sido descrita com frequência em estudos na literatura. Trata-se normalmente de uma característica relacionada ao sexo feminino ${ }^{(21)}$. Resultados semelhantes foram encontrados em estudos nacionais e internacionais conduzidos com adolescentes ${ }^{(4,22,23)}$.

Uma pesquisa realizada na cidade de Ipê/RS, com mulheres entre 18 e 56 anos de idade, registrou que $49 \%$ das mulheres classificadas com sobrepeso estavam IEP. Resultado semelhante foi encontrado em estudo conduzido na Alemanha com mulheres classificadas com sobrepeso. Na ocasião, os resultados demonstraram prevalência na insatisfação corporal( ${ }^{(24)}$. Ainda estudo realizado na cidade de Uberlândia, Minas Gerais, que avaliou mulheres entre 20 e 30 anos de idade, corrobora com os presentes achados, no qual a insatisfação com a IC ocorreu em $90 \%$ das mulheres classificadas com sobrepeso ${ }^{(25)}$.

Esses achados podem ser considerados positivos ao se interpretar que a IEP entre as participantes classificadas com sobrepeso advêm de um desejo de melhorar questões pessoais relacionadas è estética e à saúde, podendo, para tal, modificar e até mesmo assumir novos comportamentos ${ }^{(7)}$.

É importante ressaltar, entretanto, que as adolescentes com estado nutricional adequado apresentaram IM e IEP na presente pesquisa. Em estudo realizado na cidade de Pelotas, Rio Grande do Sul, com 4.235 adolescentes de 14 e 15 anos de idade, assim como no presente estudo, chama a atenção que adolescentes com peso normal apresentaram um elevo percentual de insatisfação corporal $^{(26)}$. 
Esse resultado pode estar relacionado à menor consciência corporal. Ambientes onde os adolescentes passam boa parte do seu dia podem servir como incentivadores de um estilo de vida mais saudável e ativo. Nesse sentido, a escola é um ambiente fundamental para adoção desses comportamentos. Por isso, é necessário maior esclarecimento em ambiente escolar sobre a importância da autoestima, estimulando uma maior consciência corporal. Os resultados do presente estudo demonstraram que, independente da faixa etária, a maioria das adolescentes estava IEP. Em estudo transversal, realizado com 573 adolescentes, com faixa etária entre 8 e 10 anos de idade, na cidade de Dois Irmãos e Morro Reuter, Rio Grande do Sul, os resultados encontrados foram semelhantes ao do estudo em questão ${ }^{(27)}$. Outro estudo, realizado na cidade de Gravataí, Rio Grande do Sul, com 1.442 adolescentes, com faixa etária entre 10 e 15 anos de idade, também registrou associação da IC com a faixa etária. Nesse estudo, os adolescentes entre 13 e 15 anos foram os que apresentaram maior insatisfação com a $\mathrm{IC}^{(9)}$. Os autores observaram uma tendência de mudança da insatisfação corporal, afirmando que o desejo de perder peso aumenta com a idade, enquanto o de ganhar peso diminui ${ }^{(27)}$.

Assim, a elevada prevalência de IEP identificada no presente estudo, independente da idade, pode estar diretamente relacionada ao contexto social, no qual a mídia exerce forte influência. Embora esse não seja o único fator a ser considerado, observa-se que principalmente a televisão e a internet têm contribuído na divulgação do corpo perfeito, tendo grande peso sobre a valorização do culto aos músculos e o desejo da conquista de corpos cada vez mais magros e rejuvenescidos ${ }^{(28,29)}$.

Quanto à prática de atividade física, foi registrada uma prevalência de $(43,5 \%)$ de adolescentes do sexo feminino que praticavam atividade física IEP. Corroborando com o presente estudo, em pesquisa realizada na zona rural do estado do Rio Grande do Sul, Brasil, com 510 adolescentes, os resultados registraram uma maior prevalência de insatisfação corporal entre os adolescentes que praticavam atividade física ${ }^{(30)}$.

Os benefícios decorrentes da prática regular de atividade física são bem descritos na literatura. Sabe-se que a prática de atividade física pode estar diretamente relacionada à obtenção do corpo ideal, fato que pode justificar a prevalência de meninas IEP encontradas no estudo. Muitas pessoas procuram a prática de atividade física prioritariamente na busca por estereótipos incitados pela sociedade, estando, portanto, pressionadas a manterem a aparência física dita perfeita, o que várias vezes não acontece e ocasiona em insatisfação corporal ${ }^{(3,4)}$

Assim, é importante destacar que os resultados encontrados neste estudo são preocupantes, uma vez que a insatisfação com a IC está associada com sintomas depressivos, estresse, baixa autoestima, distúrbios metabólicos, anorexia, bulimia, entre outros, tendo consequência para a saúde do individuo ${ }^{(2,3,4,9)}$. Portanto, esses resultados nos direcionam ao fato das adolescentes participantes da pesquisa estarem mais preocupadas em atingir um corpo ideal somente no intuito de satisfação pessoal, deixando de lado os demais benefícios oriundos da prática de atividade física ${ }^{(15)}$.

Algumas limitações também precisam ser consideradas. A utilização da escala de silhuetas, pois são figuras bidimensionais e não permitem a representação do individuo como um todo, o que pode implicar em falhas na representação do corpo e na distribuição da massa de gordura corporal. Além disso, a escala de silhuetas utilizada não teve sua validade testada com adolescentes brasileiros, ainda que possua validade para a população adulta e o uso desse instrumento tenha recebido boa aceitação na literatura.

Outro fator limitante foi a utilização do IMC, que, mesmo sendo uma medida bastante empregada, não deve ser o único parâmetro para a avaliação da obesidade ou massa corporal gorda, visto que não distingue de maneira adequada massa gorda e massa magra.

Pesquisas como esta são importantes por identificar grupo mais susceptíveis à insatisfação corporal em ambiente escolar, assim como por possibilitar um entendimento mais amplo de possíveis fatores associados à insatisfação corporal, proporcionando um direcionamento a ser seguido por instituições de ensino. Espera-se que os resultados apresentados e as críticas produzidas neste estudo sirvam para estimular a sistematização e publicação de informações sobre fatores comportamentais nocivos à saúde, produzindo eventualmente intervenções com objetivo de desenvolver hábitos de vida saudáveis em adolescentes.

Acrescenta-se ainda que ambientes onde o adolescente passa grande parte do seu dia são ideais para a sistematização de programas de intervenção que objetivem mudanças no estilo de vida, sendo a escola o local ideal para este tipo de intervenção por reunir periodicamente um grande número de adolescentes em seus turnos de estudo. Esperam-se ainda mais esclarecimentos em ambiente escolar sobre a importância da autoestima, estimulando uma maior satisfação com a IC.

\section{CONCLUSÃO}

Os resultados indicaram uma prevalência de adolescentes insatisfeitas com a imagem corporal (IC), em que a maioria estava insatisfeita por excesso de peso (IEP). Verificou-se associação da imagem corporal com a faixa etária e prática de atividade física, sem estar associada ao estado nutricional. 


\section{REFERÊNCIAS}

1. Alvarenga MS, Philippi ST, Lourenço BH, Sato PM. Body image dissatisfaction in felame Brazilian university studentes. J Bras Psiquiatr. 2010;59(1):44-51.

2. Poltronieria TS, Tussetb C, Gregolettoc MLO, Cremonesed C. Insatisfação com a imagem corporal e fatores associados em mulheres do sul do Brasil. Ciênc Saúde (Porto Alegre). 2016;9(3):128-34.

3. Felden EPG, Claumann GS, Sacomori C, Daronco LSE, Cardoso FL, Pelegrini A. Fatores sociodemográficos e imagem corporal em adolescentes do ensino médio. Ciênc Saúde Coletiva. 2015;20(11):3329-37.

4. Dumith SC, Menezes AMB, Bielemann RM, Petresco S, Silva ICM, Linhares RS, et al. Insatisfação corporal em adolescentes: um estudo de base populacional. Ciênc Saúde Coletiva. 2012;17(9):2499-505.

5. Reis NM, Machado Z, Pelegrini A, Boing L, Monte FCSG, Simas JPN, et al. Body image, nutritional status and symptoms of eating disorders in dancers. Rev Bras Ativ Fis Saúde. 2013;18(6):763-81.

6. Claumann GS, Pereira EF, Inácio S, Santos MC, Martins AC, Pelegrini A. Body image satisfaction in freshmen college students in physical education courses. Rev Educ Fís. 2014;25(4):575-83.

7. Mota DCL, Laus MF, Almeida SS, Costa TMB, Moreira RCM. Imagem corporal e suas relações com a atividade física e o estado nutricional em adolescentes. Rev Psicol. 2012;43(2):237-42.

8. Marques RSA, Oliveira AP, Assis MR. Prevalência de insatisfação com a imagem corporal entre estudantes de educação física. Corpus \& Cient. 2013;9(1):65-78.

9. Iepsen AM, Silva MC. Prevalência e fatores associados à insatisfação com a imagem corporal de adolescentes de escolas do Ensino Médio da zona rural da região sul do Rio Grande do Sul, 2012. Epidemiol Serv Saúde. 2014;23(2):317-25.

10. Stunkard AJ, Sorensen T, Schlusinger F. Use of the Danish Adoption Register for the study of obesity and thinness. In: Kety SS, Rowland LP, Sidman RL, Matthysse SW, editors. The genetics of neurological and psychiatric disorders. New York: Raven; 1983. p. 115-20.

11. Conde WL, Monteiro CA. Body mass index cutoff points for evaluation of nutritional status in Brazilian children and adolescents. J Pediatr. 2006;82(4):266-72.

12. World Health Organization. Physical status: the use and interpretation of anthropometry. Geneva: WHO; 1995. (Technical Report Series, 854).

13. Glaner MF, Pelegrini A, Cordoba CO, Pozzobon ME. Associação entre insatisfação com a imagem corporal e indicadores antropométricos em adolescentes. Rev Bras Educ Fís Esporte 2013;27(1):129-36.

14. Pelegrini A, Coqueiro RS, Beck CC, Ghedin KD, Lopes AS, Petroski EL. Insatisfação com a imagem corporal entre adolescentes estudantes: associação com fatores sociodemográficos e estado nutricional. Ciênc Saúde Coletiva. 2014;19(4):1201-8.

15. Santos JFS, Oliveira P, Campos AA, Pereira EO, Sousa EA. Relação entre insatisfação corporal e atividade física em adolescentes da zona urbana de Irati-PR. Rev Educ Fís. 2014;25(2):193-201.

16. Alves E, Vasconcelos FAG, Calvo MCM, Neves J. Prevalência de sintomas de anorexia nervosa e insatisfação com a imagem corporal em adolescentes do sexo feminino do município de Florianópolis, Santa Catarina, Brasil. Cad Saúde Pública. 2008;24(3):503-12.

17. McCabe MP, Ricciardelli L, Waqa G, Goundar R, Fotu K. Body image and body change strategies among adolescent males and females from Fiji, Tonga and Australia. Body Image. 2009;6(2):299-303.

18. Tiggemann M, Slater A. NetGirls: the internet, Facebook, and body image concern in adolescent girls. Int J Eat Disord. 2013;46(6):630-3.

19. Miranda VPN, Carvalho PHB, Miranda MV Júnior, Rocha JC, Ferreira MEC. Satisfação corporal de universitários de diferentes áreas de conhecimento. Cole Pesqui Educ Fís. 2012; 11(3):185-94.

20. Souza MCDFP, Souza LV, Barroso SM, Scorsolini-Comin F. Padrões alimentares e imagem corporal em mulheres frequentadoras de academia de atividade física. Psico-USF. 2013;18(3):445-54.

21. Del Ciampo LA, Del Ciampo IRL. Adolescência e imagem corporal. Adolesc Saúde 2010; 7(4):55-9. 
22. Petroski EL, Pelegrini A, Glaner MF. Motivos e prevalência de insatisfação com a imagem corporal em adolescentes. Ciênc Saúde Coletiva. 2012;17(4):1071-7.

23. Haines J, Kleinman KP, Rifas-Shiman SL, Field AE, Austin SB. Examination of shared risk and protective factors for overweight and disordered eating among adolescents. Arch Pediatr Adolesc Med. 2010;164(4):336-43.

24. Lengerke T, Mielck A, Group KS. Body weight dissatisfaction by socioeconomic status among obese, preobese and normal weight women and men: results of the cross-sectional kora augsburg population survey. BMC Public Health. 2012;12(342):2-11.

25. Curi GI, Bueno CR Júnior. Prevalência de insatisfação da imagem corporal em mulheres eutróficas com sobrepeso e obesas frequentadores de academia de ginástica. Rev Bras Obes Nutr Emagrecimento. 2011;5(23):17-23.

26. Fidelix LY, Silva DAS, Pelegrini A, Silva AF, Petroski EL. Insatisfação com a imagem corporal em adolescentes. Rev Bras Cineantropom Desempenho Hum. 2012;13(3):202-7.

27. Triches RM, Giugliani ERJ. Insatisfação corporal em escolares de dois municípios da região Sul do Brasil. Rev Nutr. 2007;20(2):119-28.

28. Vilela JEM, Lamounier JA, Dellaretti MA Filho, Barros JR Neto, Horta GM. Transtornos alimentares em escolares. J Pediatr. 2004;80(1):49-54.

29. Calado M, Lameiras M, Sepulveda AR, Rodriguez Y, Carrera MV. The mass media exposure and disordered eating behaviours in Spanish secondary students. Eur Eat Disord Rev. 2010;18(5):417-27.

30. Legnani RFS, Legnani E, Gasparotto GS, Bacil EDA, Silva MP, Campos W. Hábitos de sono e prática habitual da atividade física em escolares: uma revisão sistemática. Rev Educ Fís. 2015; 26(1):147-56.

\section{Endereço para correspondência:}

Felipe Rocha Alves

Universidade Federal do Ceará - UFC

Departamento de Medicina Clínica

Rua Costa Mendes, $1608 / 4^{\circ}$ Andar

Bairro: Rodolfo Teófilo

CEP 60430-140 - Fortaleza - CE - Brasil

E-mail: proffelipe91@hotmail.com 